\title{
Administration of metformin to increase the efficacy of chemotherapy regimen in cancers; a new look to an old drug
}

\author{
Niloufar Hooshyar $^{1 \oplus}$, Ramin Tolouian $^{2 \oplus}$, Behzad Ghasemi $^{3 \oplus}$, Nafiseh Nowrouzi $^{3 \oplus}$, Banafsheh Yalameha $^{4 \oplus}$, \\ Mohammadreza Khosravifarsani ${ }^{5^{*} \odot}$, Parto Nasri $^{6^{\odot}}$
}

${ }^{1}$ Faculty of Medicine, Isfahan University of Medical Sciences, Isfahan, Iran

${ }^{2}$ Department of Medicine, University of Arizona, Tucson, Arizona, USA

${ }^{3}$ Faculty of Pharmacy, Pécs University, Pécs, Hungary

${ }^{4}$ Department of Biochemistry, Faculty of Medicine, Lorestan University of Medical Sciences, Khorramabad, Iran

${ }^{5}$ Cancer Prevention Research Center, Isfahan University of Medical Sciences, Isfahan, Iran

${ }^{6}$ Infectious Diseases and Tropical Medicine Research Center, Isfahan University of Medical Sciences, Isfahan, Iran

\section{*Correspondence to}

Mohammadreza Khosravifarsani,

Email:drmohammadkhf@gmail.

com and Khosravifarsani@med.

mui.ac.ir

Received 14 January 2019

Accepted 8 April 2019

Published online 3 May 2019

Keywords: Chemotherapy,

Metformin, Cancer, Tumor, type

2 diabetes, Adjuvant therapy,

Chemotherapy, Malignancy

Citation: Hooshyar N, Tolouian R, Ghasemi B, Nowrouzi N, Yalameha B, Khosravifarsani M, et al. Administration of metformin to increase the efficacy of chemotherapy regimen in cancers; a new look to an old drug. Immunopathol Persa. 2019;5(1):e11. DOI:10.15171/ ipp.2019.11

\begin{abstract}
Chemotherapeutic agents are still the major and most common therapy to prevent tumor growth. These drugs have various adverse effects in different organs in addition to systemic effects. Finding more specific and effective drugs or new adjuvant therapeutic substance is needed to improve the success rate. Several studies have proposed the possible mechanisms of anti-neoplastic of metformin, however, its exact mechanism is still obscure. The suggested mechanisms are; alteration in the host metabolic environment after administration of metformin, such as decreases in insulin-dependent stimulation of tumor growth or direct effect on cancer cells, such as the impact on adenosine monophosphate (AMP)-activated protein kinase signaling pathway. Metformin, as an adjuvant therapy, synergistically exerts growth inhibitory effects against cell growth and can induce cell apoptosis in an animal model. Adding metformin to the chemotherapy regimen may reduce resistance and enhance therapeutic efficacy.
\end{abstract}

\section{Introduction}

Malignancy is still one of the most challenging illnesses to treat despite much progress in recent years. Mortality rate has been reduced only marginally due to the intrinsic behavior of tumors, heterogeneous nature of malignancy and different degrees of response to treatment $(1,2)$.

Cancer cells are recognized and defined by their high production rate and resistance to apoptotic cell death. Chemotherapeutic agents are still the major and most common therapy to prevent tumor growth. The side effects of chemotherapy agents have negative impacts on the quality of life. Meanwhile, malignant cells frequently became resistant to chemotherapy, which results in cancer reappearance or metastasis. Chemotherapeutic agents express antitumor properties by preventing cancer cell growth through cell cycle arrest and stimulation of cellular apoptosis. Progression to advance stages or metastasis is a common

\section{Key point}

Numerous epidemiologic studies show the ameliorating impact of administration of metformin to chemotherapy regimens on the remission of cancers.

scenario in the current chemotherapy era. Chemotherapeutic drugs have various adverse effects in different organs in addition to systemic effects. Common systemic adverse effects include loss of appetite, fatigue, infertility, nausea, vomiting, and hair loss. These side effects may lessen with reducing the dosage of chemotherapeutic agents. However, it may diminish the effectiveness of chemotherapeutic drugs to inhibit tumor growth. Therefore, finding more specific and effective drugs or new adjuvant therapeutic substance is needed to improve the success rate $(3,4)$. Mammalian cell death occurs by various mechanisms, including apoptosis, necrosis and

\footnotetext{
Copyright (C) 2019 The Author(s); Published by Nickan Research Institute. This is an open-access article distributed under the terms of the Creative Commons Attribution License (http://creativecommons.org/licenses/by/4.0), which permits unrestricted use, distribution, and reproduction in any medium, provided the original work is properly cited.
} 
autophagy. Autophagy is a catabolic activity which leads to modification of cytoplasmic materials. This process leads to organelle impairment and accumulation of abnormal proteins. Autophagy is activated by different mechanisms and occurs in various malignant cells. Metformin, activates the autophagy pathway and hence, might be a candidate to use as an adjuvant therapy in cancer treatment $(5,6)$.

\section{Materials and Methods}

Web of Science, directory of open access journals (DOAJ), PubMed/Medline, EBSCO, Embase, Scopus, and Google Scholar were searched using keywords of chemotherapy, metformin, cancer, tumor, type 2 diabetes, adjuvant therapy, malignancy and adenosine monophosphate (AMP)-activated protein kinase

\section{Metformin in type 2 diabetes}

Metformin is the first line of antidiabetic agent for diabetes mellitus type II. Metformin is a relatively cheap and well tolerated drug. It has a low risk of hypoglycemia and advantage of countering insulin resistance. Approximately 120 million prescriptions are filled globally each yearbecause of its positive risk-benefit profile (7).

The pathophysiology of antitumor effects of metformin Several studies have proposed the possible mechanisms of anti-neoplastic of metformin. However, its exact mechanism is still obscure. The suggested mechanisms are alterations in the host metabolic environment after administration of metformin, such as decreases in insulindependent stimulation of tumor growth. Metformin may also have a direct effect on cancer cells. Metformin reduces insulin/insulin-like growth factor 1 (IGF-1), insulin receptor and/or IGF-1 receptors. In diabetes, increasing insulin and IGF-1 receptor or hyperglycemia, have been perceived to provoke mitosis and tumor growth. Metformin opposes these signaling routes and potentially might be beneficial in reducing multidrug resistant cancer and prevention of tumor metastasis $(8,9)$.

Metformin also inhibits the glycolysis and perturbation of electron transport chain in the tricarboxylic acid cycle. This phenomenon decreases ATP creation and depletion of cellular aspartate, which is vital for nucleotide synthesis. This pathway may explain the anticancer activity of metformin $(10,11)$.

Metformin increases the level of AMP and prevents adenylate cyclase activity and mTORC1 signaling. Metformin causes a temporary reduction in cellular energy status, which causes a total decrease in ATP using processes. In proliferating cells like malignancies, this phenomenon may stimulate a cytostatic condition which will reduce the proliferation of cells. In fact, malignant cells in the presence of metformin cannot balance the reduced energy condition, thus cells will go toward apoptosis (1214).
Adenosine monophosphate -activated protein kinase (AMPK)

AMP-activated protein kinase is a heterotrimeric complex designed by three subunits; alpha, beta and gamma. The alpha subunit is accountable for the catalytic action while the beta and gamma subunits are related to the changes of the kinase activity.

To stimulate its kinase activity, AMP-activated protein kinase should be phosphorylated on the residual alpha subunit by liver kinase B1 (LKB1). This phosphorylation is delicately moderated by allosteric modifications. Numerous studies have shown that administration of metformin results in a decrease in ATP in hepatic cells and a reduction in the $\mathrm{ATP} / \mathrm{ADP}$ ratio, leading to a drop in cellular bioenergetics.

Metformin also causes stimulation of intracellular hepatic enzyme of AMP-activated protein kinase which leads to an increase in beta-oxidation, or autophagy. This pathway is required for the regulation of cell proliferation and energy metabolism.

AMP-activated protein kinase is an important moderator of various processes within the cells. The dysregulation of AMPK accompanies numerous pathological situations, such as diabetes, neurodegeneration, malignancy, and heart disease. In fact, when AMPK is triggered, incipient malignant cell death may happen in the absence of sufficient energy stores. Various conditions can stimulate AMPK in the body, such as calorie restriction, which depresses cellular energy supplies and triggers AMPK. This is the reason that calorie constraint has been detected to diminish malignancy occurrence and augment cancer cell death in animal models (15-18).

Metformin also hinders mitochondrial complex I activation in various malignant cells, which consequently reduces tumorigenesis. In addition, metformin provokes cells to preserve or reduce energy or may halt abnormal cell growth completely.

Metformin enhances the effectiveness of cytotoxic $\mathrm{T}$ cell via programmed death-ligand 1 (PD-L1) on cancer cells. The mechanism by which metformin enhances the cytotoxicity of cancer drugs is dependent on the presentation of the energy sensor kinase AMPK in malignant cells.

Metformin activates AMPK, which phosphorylates synthesized PD-L1 in the endoplasmic reticulum lumen. This phosphorylation results in abnormal $\mathrm{N}$-glycosylation of PD-L1 and prevents its development within the Golgi apparatus and degradation in endoplasmic reticulum. Therefore, metformin causes malignant cells to be more susceptible to cytotoxic T cells (19-22).

\section{Metformin and mitochondria}

Metformin attracts and aggregates in mitochondria because of its positive molecular charge. Subsequently, it prevents the complex I of the mitochondrial electron transport 
chain, leading to a decline in cellular ATP and a rise in both AMP/ATP and ADP/ATP ratios. Low energy level activates AMPK, a key energy sensor, which is implicated in various activities within the cells to reestablish energy homeostasis (23-25).

Activation of AMPK directs the cells toward an antiproliferative capability that leads to inhibition of tumor expansion. (26-28). Moreover, metformin impedes the mitochondrial glycerophosphate dehydrogenase (29-31).

\section{Discussion}

Iliopoulos et al showed that metformin prevents the growth of breast cancer cells in animal models. It has been observed that the co-administration of metformin and doxorubicin enhances the proportion of cancer regression and delay of relapse, in comparison to treatment with doxorubicin alone (32). Another retrospective study demonstrated a higher frequency of complete remission of breast cancer in diabetic patients on metformin compared to diabetic individuals taking other antidiabetic agents while undergoing chemotherapy (33). Xu et al demonstrated that metformin inactivates stromal fibroblasts in ovarian cancer and reduced fibroblastfacilitated tumor growth in their models. They concluded that metformin prevents interleukin 6 secretion through inhibiting NF- $\mathrm{BB}$ signaling, the upstream controller of stromal inflammation. It is postulated that an alternative modality in targeting stromal inflammation by a potential plan of combination therapy with metformin may ameliorate the chemo-sensitivity in ovarian cancer (34). A meta-analysis/systematic review by Coyle et al, suggested that metformin could be a suitable adjuvant therapy in prostate and colorectal malignancies, especially in individuals who received radical radiotherapy (35). Xin et al conducted a study on MCF-7 breast cancer cells. They demonstrate that the combination of formononetin and metformin potentiate cell growth inhibition, and the initiation of apoptosis in MCF-7 cells mediated by the ERK1/2 signaling pathway (36). Jafari-Gharabaghlou et al have also demonstrated the anti-proliferative properties of metformin and phenformin in combinational therapy, against breast cancer cells. In their experiment, the combination of metformin and phenformin enhanced the efficacy of treatment (37). Hamedi et al conducted a randomized clinical trial on individuals with primary ovarian cancer with a 4-year follow-up period. They divided their patients into two study groups. The study arm received $500 \mathrm{mg}$ metformin three times a day in addition to a standard chemotherapy regimen while the control arm received only the standard chemotherapeutic drugs after surgery.

This study demonstrated that the addition of metformin to the chemotherapy regimen of patients undergoing surgery for ovarian cancer can improve the survival (48 months vs. 25.7 months in the control group) and declining the rate of relapse ( $13.3 \%$ vs. $67.5 \%$ in the control group).
It is possible that metformin intensifies the cytotoxic effects of chemotherapy in tumor cells and other tissues by modifying various gene presentation routes (38). In a recent multicenter randomized phase II trial, Narita et al compared the efficacy and safety of chemotherapy regimens by adding metformin in patients with resected pancreatic ductal adenocarcinoma. Metformin is administrated in conjunction with chemotherapy for two years. It has been shown that 2-year-survival rate improved from $65 \%$ in the control group to $78 \%$ in the study group. It also demonstrated an improvement of the 5 -year survival rate to $44.1 \%$. This epidemiologic study suggests that metformin may have a favorable impact on the prognosis of pancreatic ductal adenocarcinoma (39). Kuo et al conducted a study on head and neck squamous cell carcinoma. They compared the combination of metformin to cisplatin treatment versus cisplatin alone. They measured cell proliferation level by MTS assays. This observation demonstrated that metformin protects head and neck squamous cell cancer against cisplatin in vitro (40). Sharma et al demonstrated that metformin notably diminished cholesterol content in breast cancer cells with parallel inhibition of several cholesterol regulatory genes. The authors concluded that anticancer activity through decreasing cholesterol content in breast cancer cells is one of the anticancer mechanisms of metformin (41). Chen et al observed that proliferation of gastric cancer cells is reduced by exposure to metformin. The suppressive impact of metformin on gastric cancer is mediated through stimulating calmodulin-like protein 3 secretion from tumor-associated fibroblasts (42).

Mitochondrial glycerol 3-phosphate dehydrogenase is a main provider of electrons to the transport chain in mitochondria. This enzyme is necessary for gluconeogenesis. Mitochondrial glycerol 3-phosphate dehydrogenase is also the main enzyme linking oxidative phosphorylation and glycolysis. This enzyme is a target of metformin in the liver. Thakur et al have demonstrated that mitochondrial glycerol 3-phosphate dehydrogenase is overexpressed in thyroid cancer. Metformin decreases this enzyme expression, inhibits mitochondrial respiration and stops the shift of cancer cell metabolism toward glycolysis (43).

\section{Conclusion}

Administration of metformin as an adjuvant therapy synergistically exerts growth inhibitory effects against cell growth and can induce cell apoptosis. Adding metformin to the chemotherapy regimen may reduce resistance and enhance therapeutic efficacy.

\section{Authors' contribution}

$\mathrm{NH}, \mathrm{BG}, \mathrm{NN}, \mathrm{PN}$ and BY wrote the paper. RT edited the manuscript. All authors read and signed the final paper.

Conflicts of interest

The authors declare no conflict of interest. 
Ethical considerations

Ethical issues (including plagiarism, data fabrication, double publication) have been completely observed by the authors.

\section{Funding/Support}

None.

\section{References}

1. Riaz MA, Sak A, Erol YB, Groneberg M, Thomale J, Stuschke $M$. Metformin enhances the radiosensitizing effect of cisplatin in non-small cell lung cancer cell lines with different cisplatin sensitivities. Sci Rep. 2019; 9:1282. doi: 10.1038/s41598-01838004-5.

2. Yousef M, Tsiani E. Metformin in Lung Cancer: Review of in Vitro and in Vivo Animal Studies. Cancers (Basel). 2017; 9: 45.

3. Zhang HH, Guo XL.Combinational strategies of metformin and chemotherapy in cancers. Cancer Chemother Pharmacol. 2016; 78:13-26. doi: 10.1007/s00280-016-3037-3.

4. Courtois S, Durán RV, Giraud J, Sifré E, Izotte J, Mégraud F, et al. Metformin targets gastric cancer stem cells. Eur J Cancer. 2017; 84:193-201. doi: 10.1016/j.ejca.2017.07.020.

5. Zhuo Z, Wang A, Yu H. Metformin targeting autophagy overcomes progesterone resistance in endometrial carcinoma. Arch Gynecol Obstet. 2016; 294:1055-1061.

6. Saini N, Yang X. Metformin as an anti-cancer agent: actions and mechanisms targeting cancer stem cells. Acta Biochim Biophys Sin (Shanghai). 2018; 50:133-143. doi: 10.1093/abbs/ gmx106.

7. Hostalek U, Gwilt M, Hildemann S. Therapeutic use of metformin in prediabetes and diabetes prevention. Drugs. 2015; 75:1071-94. doi: 10.1007/s40265-015-0416-8.

8. Grimberg A. Mechanisms by which IGF-I may promote cancer. Cancer Biol Ther. 2003;2:630-5.

9. Lei Y, Yi Y, Liu Y, Liu X, Keller ET, Qian CN, et al. Metformin targets multiple signaling pathways in cancer. Chin J Cancer. 2017;36:17. doi: 10.1186/s40880-017-0184-9.

10. Marrone KA, Zhou X, Forde PM, Purtell M, Brahmer JR, Hann $\mathrm{CL}$ et al. A randomized phase II study of metformin plus paclitaxel/carboplatin/bevacizumab in patients with chemotherapy-naïve advanced or metastatic nonsquamous non-small cell lung cancer. Oncologist. 2018;23:859-865. doi: 10.1634/theoncologist.2017-0465.

11. Bridges $H R$, Jones AJY, Pollak MN, Hirst J. Effects of metformin and other biguanides on oxidative phosphorylation in mitochondria. Biochem J. 2014;462:475-487. doi: 10.1042/ BJ20140620.

12. Andrzejewski S, Siegel PM, St-Pierre J. Metabolic profiles associated with metformin efficacy in cancer. Front Endocrinol (Lausanne). 2018;9:372. doi: 10.3389/fendo.2018.00372.

13. Han J, Wysham WZ, Zhong Y, Guo H, Zhang L, Malloy KM, et al. Increased efficacy of metformin corresponds to differential metabolic effects in the ovarian tumors from obese versus lean mice.Oncotarget. 2017;8:110965-82. doi: 10.18632/ oncotarget.20754.

14. Wahdan-Alaswad R, Fan Z, Edgerton SM, Liu B, Deng XS, Arnadottir SS, et al. Glucose promotes breast cancer aggression and reduces metformin efficacy. Cell Cycle. 2013;12:3759-69. doi: 10.4161/cc.26641.

15. Chiang CF, Chao TT, Su YF, Hsu CC, Chien CY, Chiu KC, et al.Metformin-treated cancer cells modulate macrophage polarization through AMPK-NF-KB signaling. Oncotarget. 2017; 8:20706-18. doi: 10.18632/oncotarget.14982.

16. Li W, Saud SM, Young MR, Chen G, Hua B.Targeting AMPK for cancer prevention and treatment. Oncotarget. 2015;6:736578. doi:10.18632/oncotarget.3629.

17. Umezawa S, Higurashi T, Nakajima A. AMPK: Therapeutic
Target for Diabetes and Cancer Prevention.Curr Pharm Des. 2017; 23:3629-44. doi: 10.2174/0929867324666170713150 440.

18. Gwak H, Kim Y, An H, Dhanasekaran DN, Song YS. Metformin induces degradation of cyclin D1 via AMPK/GSK3 $\beta$ axis in ovarian cancer. Mol Carcinog. 2017;56:349-358. doi: 10.1002/mc.22498.

19. Cha JH, Yang WH, Xia W, Wei Y, Chan LC, Lim SO. Metformin Promotes Antitumor Immunity via Endoplasmic-ReticulumAssociated Degradation of PD-L1. Mol Cell. 2018;71:606620.e7. doi: 10.1016/j.molcel.2018.07.030.

20. Choi YK, Park KG. Metabolic Roles of AMPK and Metformin in Cancer Cells. Mol Cells. 2013;36:279-87.doi: 10.1007/ s10059-013-0169-8.

21. Li W, Saud SM, Young MR, Chen G, Hua B. Targeting AMPK for cancer prevention and treatment. Oncotarget. 2015; 6: 7365-7378. doi: 10.18632/oncotarget.3629.

22. Duan Q, Song P, DingY, Zou MH. Activation of AMP-activated protein kinase by metformin ablates angiotensin II-induced endoplasmic reticulum stress and hypertension in mice in vivo. Br J Pharmacol. 2017;174:2140-2151. doi: 10.1111/ bph. 13833 .

23. Curry DW, Stutz B, Andrews ZB, Elsworth JD. Targeting AMPK Signaling as a Neuroprotective Strategy in Parkinson's disease. J Parkinsons Dis. 2018;8:161-81. doi: 10.3233/JPD-171296.

24. Hongying An, Ling He. Current Understanding of Metformin Effect on the Control of Hyperglycemia in Diabetes. J Endocrinol. 2016;228:97-106. doi: 10.1530/JOE-15-0447.

25. Stephenne X, Foretz M, Taleux N, van der Zon GC, Sokal E, Hue $L$, et al. Metformin activates AMP-activated protein kinase in primary human hepatocytes by decreasing cellular energy status. Diabetologia. 2011;54:3101-10. doi: 10.1007/s00125011-2311-5.

26. Mihaylova MM, Shaw RJ. The AMP-activated protein kinase (AMPK) signaling pathway coordinates cell growth, autophagy, \& metabolism. Nat Cell Biol. 2011;13:1016-23. doi: 10.1038/ ncb2329.

27. Howell JJ, Hellberg K, Turner M, Talbott G, Kolar MJ, Ross DS, et al. Metformin inhibits hepatic mTORC1 signaling via dose-dependent mechanisms involving AMPK and the TSC complex. Cell Metab. 2017;25:463-71. doi: 10.1016/j. cmet.2016.12.009.

28. Dungan CM, Li Z, Wright DC, Williamson DL. MTORC1 signaling is unaffected by metformin treatment in aged skeletal muscle. Muscle Nerve. 2016;53:107-17. doi: 10.1002/ mus. 24698

29. Kasznicki J, Sliwinska A, Drzewoski J. Metformin in cancer prevention and therapy. Ann Transl Med. 2014;2:57. doi: 10.3978/j.issn.2305-5839.2014.06.01

30. Soliman GA, Steenson SM, Etekpo AH. Effects of Metformin and a Mammalian Target of Rapamycin (mTOR) ATPCompetitive Inhibitor on Targeted Metabolomics in Pancreatic Cancer Cell Line. Metabolomics (Los Angel). 2016;6:183. doi: 10.4172/2153-0769.1000183.

31. Cifarelli V, Lashinger LM, Devlin KL, Dunlap SM, Huang J, Kaaks $\mathrm{R}$, et al. Metformin and rapamycin reduce pancreatic cancer growth in obese prediabetic mice by distinct microRNA-regulated mechanisms. Diabetes. 2015;64:163242. doi: $10.2337 / \mathrm{db} 14-1132$.

32. Iliopoulos D, Hirsch HA, Struhl K. Metformin decreases the dose of chemotherapy for prolonging tumor remission in mouse xenografts involving multiple cancer cell types. Cancer Res. 2011;71:3196-201. doi: 10.1158/0008-5472.CAN-103471.

33. Jiralerspong S, Palla SL, Giordano SH, Meric-Bernstam F, Liedtke C, Barnett CM, et al. Metformin and pathologic 
complete responses to neoadjuvant chemotherapy in diabetic patients with breast cancer. J Clin Oncol 2009; 27:3297-3302. doi: 10.1200/JCO.2009.19.6410.

34. Xu S, Yang Z, Jin P, Yang X, Li X, Wei X, et al. Metformin suppresses tumor progression by inactivating stromal fibroblasts in ovarian cancer. Mol Cancer Ther. 2018;17:1291302. doi: 10.1158/1535-7163.MCT-17-0927.

35. Coyle C, Cafferty FH, Vale C, Langley RE. Metformin as an adjuvant treatment for cancer: a systematic review and metaanalysis. Ann Oncol. 2016;27:2184-95. doi: 10.1093/annonc/ mdw410.

36. Xin M, Wang Y, Ren Q, Guo Y. Formononetin and metformin act synergistically to inhibit growth of MCF-7 breast cancer cells in vitro. Biomed Pharmacother. 2019;109:2084-9. doi: 10.1016/j.biopha.2018.09.033.

37. Jafari-Gharabaghlou D, Pilehvar-Soltanahmadi Y, Dadashpour M, Mota A, Vafajouy-Jamshidi S, Faramarzi L, et al. Combination of metformin and phenformin synergistically inhibits proliferation and hTERT expression in human breast cancer cells. Iran J Basic Med Sci. 2018; 21:1167-1173. doi: 10.22038/IJBMS.2018.30460.7345.

38. Hamedi B, Khalili A, Roozmeh S, Namazi G, Saraf Z. Combination of metformin and chemotherapy decreases the recurrence rates of epithelial ovarian cancers: a randomized clinical trial. Int J Cancer Manag. 2018;11:e11621. doi: 10.5812/ijcm.11621.
39. Narita M, Furukawa M, Sakamoto I, Itoh Y, Nakano H, Naka T, et al. Adjuvant chemotherapy of S-1 versus S-1 plus metformin for resected pancreatic cancer: A multicenter and randomized phase II trial. J Clin Oncol. 2019;37. DOI:10.1200/ JCO.2019.37.4_suppl.TPS474.

40. Kuo SZ, Honda CO, Li WT, Honda TK, Kim E, Altuna X, et al . Metformin results in diametrically opposed effects by targeting non-stem cancer cells but protecting cancer stem cells in head and neck squamous cell carcinoma. Int J Mol Sci. 2019;20: E193. doi: 10.3390/ijms20010193.

41. Sharma A, Bandyopadhayaya S, Chowdhury K, Sharma T, Maheshwari R, Das A, et al. Metformin exhibited anticancer activity by lowering cellular cholesterol content in breast cancer cells. PLoS One. 2019;14:e209435. doi: 10.1371/ journal.pone.0209435.

42. Chen G, Yu C, Tang Z, Liu S, An F, Zhu J, et al. Metformin suppresses gastric cancer progression through calmodulin-like protein 3 secreted from tumor-associated fibroblasts. Oncol Rep. 2019;41:405-14. doi: 10.3892/or.2018.6783.

43. Thakur S, Daley B, Gaskins K, Vasko VV, Boufraqech M, Patel $\mathrm{D}$, et al. Metformin targets mitochondrial glycerophosphate dehydrogenase to control rate of oxidative phosphorylation and growth of thyroid cancer in vitro and in vivo. Clin Cancer Res. 2018;24:4030-43. doi: 10.1158/1078-0432.CCR-173167. 